\title{
Avenir du système de santé et tarifs de demain
}

\author{
Quelle place attribuer à l'Etat dans le système de santé? Quelle est l'utilité des plans \\ directeurs? Peut-on redonner de l'élan à la primauté des contrats? Et quel sera le \\ paysage tarifaire après SwissDRG et TARMED? Ce sont là quelques-unes des ques- \\ tions débattues par les nombreux participants à la séance d'information de la FMH \\ «L'avenir du système de santé», avec Thomas Christen, conseiller personnel du conseiller \\ fédéral Alain Berset, et Heinz Locher, Dr ès sc. écon., économiste de la santé.
}

\section{Beatrix Meyer}

Cheffe de la division Tarifs les médecins hospitaliers et économie de la santé pour

\begin{abstract}
S'alimenter à l'étable de l'Etat?
D'entrée de jeu, H. Locher souligne non sans crainte que la plupart des acteurs du système de santé n'ont pas assez conscience du rôle de la réglementation politique. A ses yeux, il existe aujourd'hui une tendance indéniable d'un grand nombre à vouloir profiter de «l'étable de l'Etat», pour reprendre les termes du célèbre économiste Friedrich A. Hayek. Il précise cependant que, pour lui, il ne s'agit pas d'abolir toute réglementation mais plutôt de se poser la question de la réglementation: combien en faut-il et comment
\end{abstract}

\section{La neutralité des coûts condamne les partenaires tarifaires au désaccord.}

Correspondance:

FMH

Frohburgstrasse 15

CH-4600 Olten

Tél. 0313591111

Fax 0313591112

tarife.spital[at]fmh.ch
l'Etat peut-il créer des conditions-cadres optimales? La réponse ne viendra pas des plans directeurs. A des programmes étatiques qu'il réprouve, il préfère opposer des objectifs clairement définis dans le but de laisser suffisamment d'espace aux acteurs pour mettre en place des projets-pilotes et créer une dynamique des «systèmes évolutifs». A l'opposé, Th. Christen estime que le système de santé est intrinsèquement réglementé et que, de ce fait, soulever la question fondamentale du «marché versus Etat» constitue une perte de temps. Au mieux, cette réflexion ne produirait que des fossés idéologiques sans profiter à la Suisse. Selon lui, il n'y a pas de «ou... ou», mais seulement un «et... et».

\section{Revitalisation de la primauté des contrats}

A quel point et jusqu'à quand la Confédération jouera-t-elle un rôle de réglementation dans le domaine tarifaire? Th. Christen renvoie à la compétence subsidiaire de la Confédération, qui intervient uniquement si les partenaires tarifaires ne parviennent pas à s'entendre sur la révision d'une structure tarifaire qui n'est plus adaptée. A cet égard, il souligne expressément que le Département fédéral de l'inté- rieur n'a aucun intérêt à assumer le rôle des partenaires pour l'élaboration de structures tarifaires. Aujourd'hui, dans le domaine ambulatoire, la Confédération exerce sa compétence subsidiaire uniquement durant la durée nécessaire aux partenaires pour se mettre d'accord sur une structure tarifaire. Dès lors, la question se pose de savoir si les négociations doivent absolument inclure tous les partenaires tarifaires sans exception ou si une partie des associations professionnelles, p. ex. curafutura chez les assureurs, suffit. Pour Th. Christen, il est évident que tous les partenaires doivent être consultés lors d'un changement substantiel de la structure tarifaire.

Dans le contexte actuel, la recherche d'un compromis est-elle réaliste au regard des intérêts divergents? Selon H. Locher, le maintien de la neutralité des coûts, tel qu'il est formulé, mine les conditionscadres et les négociations, et ne laisse aucune place à un accord entre tous les partenaires tarifaires. Toute tentative est vouée à l'échec. Dans ce contexte, un retour crédible et rapide à des contrats de partenariat semble nécessaire pour mettre un terme à l'étati-

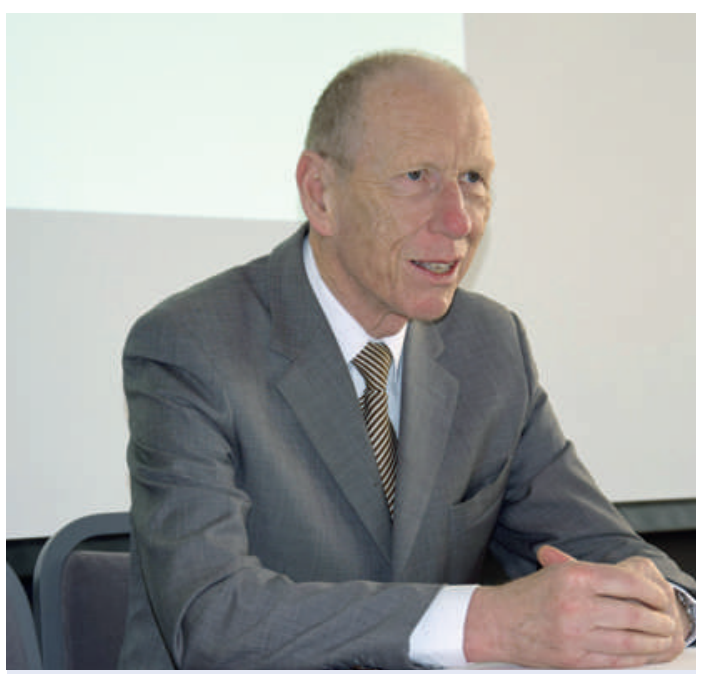

Pour H. Locher, le but premier est de fournir des prestations de qualité élevée avec efficacité et non de se focaliser sur les économies de coûts. 


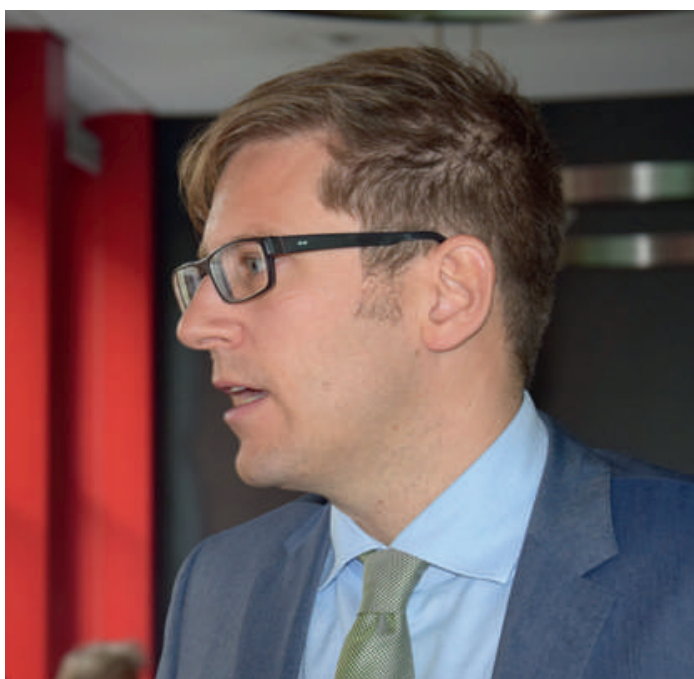

Pour Th. Christen, il est évident que tous les partenaires doivent être consultés lors d'un changement substantiel de la structure tarifaire.

sation rampante et inexorable de notre système de santé. Par ailleurs, H. Locher évoque la situation problématique des cantons qui sont désormais parties tarifaires dans le domaine hospitalier sans qu'aucune réglementation formelle n'existe.

\section{Retour aux contrats de partenariat pour arrêter l'étatisation rampante.}

\section{Modèles tarifaires de demain}

Quel sera le paysage tarifaire après SwissDRG et TARMED? H. Locher voit des tarifs qui se structurent autour de critères de qualité et encouragent tant la collaboration entre les fournisseurs de prestations qu'un emploi efficace des ressources. Pour illustrer sa vision, il présente différents modèles tarifaires basés,

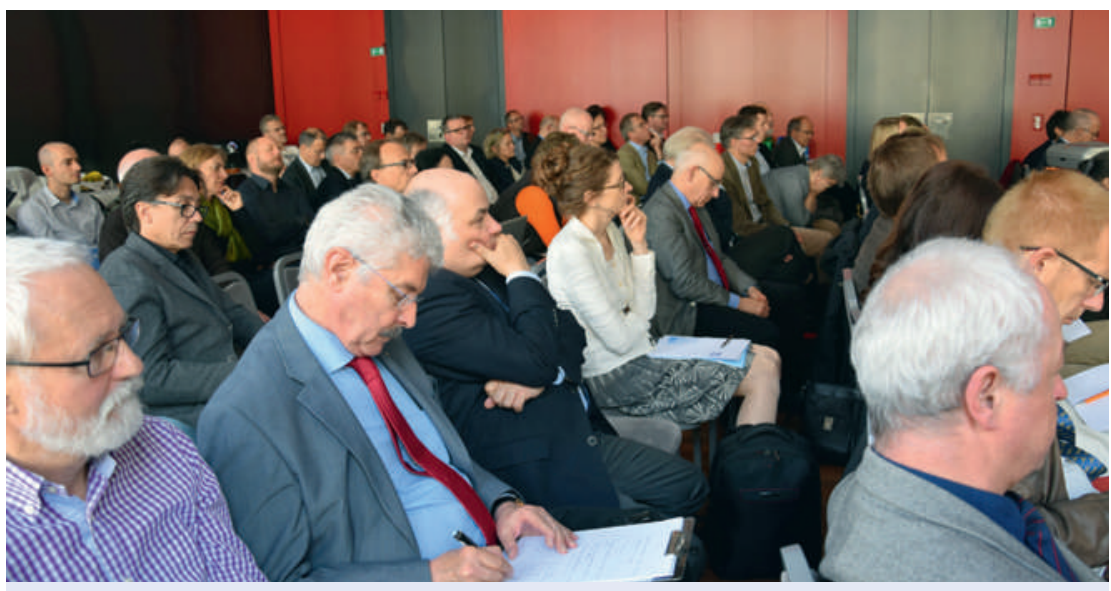

Quel sera le paysage tarifaire après SwissDRG et TARMED? De nombreux participants se sont intéressés aux réponses à cette question lors de la séance d'information de la FMH. entre autres, sur un système de forfaits complexes permettant une tarification globale, ainsi que les effets incitatifs qu'ils induisent. A titre d'exemple, il explique qu'il pourrait s'agir d'un forfait par cas du séjour hospitalier, incluant l'ensemble des prestations fournies par tous les prestataires jusqu'au terme d'une période de traitement. Il cite notamment l'exemple de Stockholm où des expériences concluantes ont été acquises en lien avec des forfaits complexes pour les prothèses de la hanche et du genou. Par ailleurs, H. Locher trouve que la décision de la caisse-maladie Sanitas de ne plus imposer de demande de garantie pour la prise en charge de la réadaptation lors de tableaux cliniques évidents va dans la bonne direction. En revanche, Th. Christen est plutôt réticent quant à l'introduction de forfaits complexes ou de changements fondamentaux. Il rappelle que la Suisse n'a pas besoin de projets de réforme importants en politique de la santé et que ceux-ci ne sont de toute façon pas susceptibles de réunir la majorité. Bien noté par les experts internationaux, le système de santé suisse donne entière satisfaction à la population. Cependant, rien n'empêche d'élaborer une stratégie pertinente pour corriger les faiblesses du système existant et relever les défis à venir.

\section{Qu'en est-il du potentiel d'économie?}

Pour Th. Christen, la stratégie Santé 2020 du Conseil fédéral marque le début et non le terme d'un processus; elle doit être concrétisée par tous les acteurs de la santé. Par ailleurs, Th. Christen remercie expressément la FMH pour sa prise de position détaillée [1] à ce sujet. Il relativise toutefois quelque peu le potentiel d'économie mentionné dans ce papier stratégique, à savoir $20 \%$ sur les prestations obligatoires. En effet, s'il est possible et nécessaire de faire des économies grâce à une augmentation de l'efficacité, le chiffre de $20 \%$ avancé et repris d'anciens rapports ne constitue pas l'objectif principal. Pour H. Locher, le but premier est de fournir des prestations de qualité élevée avec efficacité et non de se focaliser sur les économies de coûts. Il s'agit notamment d'éviter les variations régionales non justifiées, à l'image de celles mises en évidence par la recherche sur les soins. Pour conclure, il ajoute que l'environnement de la santé publique en Suisse serait tout à fait compatible avec des conditions-cadres permettant l'éclosion d'un système de santé durable, axé sur les valeurs économiques.

1 Wille N, Bütikofer A, Schlup J. «Santé 2020»: une vision viable de notre système de santé? Bull Méd Suisses. 2014;95(11):423-6.

\section{Informations supplémentaires}

Vous trouverez tous les exposés de la séance d'information de la FMH du 13 mai 2014 sur le site www.fmh.ch $\rightarrow$ Tarifs hospitaliers $\rightarrow$ Publications $\rightarrow$ L'avenir du système de santé. 